\title{
REPRESENTATIONS OF RANK ONE ALGEBRAIC MONOIDS
}

\author{
by LEX E. RENNER
}

(Received 4 February, 1987)

0. Introduction. One of the fundamental results of representation theory is the identification of the irreducible representations of a semisimple group by their dominant weights [3]. The purpose of this paper is to establish similar results for a class of reductive algebraic monoids.

Let $k$ be an algebraically closed field. An algebraic monoid is an affine algebraic variety $M$ defined over $k$, together with an associative morphism $m: M \times M \rightarrow M$ and a two-sided unit $1 \in M$ for $m$.

In [5] the set of normal, algebraic monoids with unit group $G=S l_{2}(k) \times k^{*}, G l_{2}(k)$ or $P G l_{2}(k) \times k^{*}$ is determined numerically. That construction, however, does not yield directly any irreducible representations of these monoids. In this paper I produce a complete list (see 3.7 and 3.11). This list is fundamental for studying the relationship (in general) of irreducible representations to the system of idempotents of closely related monoids [4].

1. Preliminaries. Let $M$ be an algebraic monoid, and let

$$
G=G(M)=\left\{x \in M \mid x^{-1} \in M\right\} .
$$

Then $G \subseteq M$ is an affine, open, algebraic subgroup. If $M$ is irreducible then $\bar{G}=M$ (Zariski closure). If $T \subseteq G$ is a maximal torus, then $\bar{T} \subseteq M$ is a maximal, irreducible, closed $D$-submonoid. $\bar{T}$ is determined to within an isomorphism by the commutative monoid

$$
X(\bar{T})=\{\chi \in X(T) \mid \chi \text { extends to } \bar{\chi}: \bar{T} \rightarrow k\} .
$$

A rational representation of $\bar{T}$ is simply an $X(\bar{T})$-graded vector space over $k$. See [5] for details and references.

An irreducible, algebraic monoid $M$ is reductive if $G(M)$ is a reductive group.

2. Representations of $S l_{2}(k) \times k^{*}$. This section is a summary of some of the basic properties of irreducible representations of $S l_{2}(k) \times k^{*}$.

Let $k, l \in \mathbb{N}$. Then there exists a representation

$$
\tau_{l}: S l_{2}(k) \rightarrow G l_{l+1}(k) .
$$

$\tau_{l}$ is the $l$-th symmetric power of the canonical representation $S l_{2}(k) \subseteq G l_{2}(k)$. Note that $\tau_{l}$ is not in general irreducible. We thus define for each $l \in \mathbb{N}, k \in \mathbb{Z}$,

by

$$
\rho_{k, l}: S l_{2}(k) \times k^{*} \rightarrow G l_{l+1}(k)=G l(V(k, l))
$$

$$
\rho_{k, l}(x, t)=\left(\operatorname{diag}\left[t^{k}, \ldots, t^{k}\right]\right) \tau_{l}(x) .
$$

Glasgow Math. J. 30 (1988) 237-241. 
Let $T=k^{*} \times k^{*} \subseteq S l_{2}(k) \times k^{*}$ be a maximal torus and let

$$
\begin{aligned}
& x=p_{2}: T \rightarrow k^{*}, \\
& y=p_{1}: T \rightarrow k^{*} .
\end{aligned}
$$

With respect to the basis $\{x, y\} \subseteq X(T)$, we obtain the following weight decomposition of $V=V(k, l)$ :

$$
V=V_{k x+l y} \oplus V_{k x+(l-2) y} \oplus \ldots \oplus V_{k x-l y} .
$$

Thus, if $\overline{\rho_{k, l}(T)}=Z$ is the Zariski closure of $\rho_{k, l}(T)$ in $\operatorname{End}(V)$, then

$$
X(Z)=\left\langle x^{k} y^{l}, x^{k} y^{l-2}, \ldots, x^{k} y^{-l}\right\rangle,
$$

the submonoid of $X\left(\rho_{k, l}(T)\right)$ generated by $\left\{x^{k} y^{l}, \ldots, x^{k} y^{-l}\right\}$. (We are using both additive and multiplicative notation for characters.) Let

$$
W(k, l)=\{k x+l y, \ldots, k x-l y\} .
$$

2.1. Remark. We have canonical morphisms

$$
m: S l_{2}(k) \times k^{*} \rightarrow G l_{2}(k) \text { and } c: S l_{2}(k) \times k^{*} \rightarrow P G l_{2}(k) \times k^{*},
$$

both of degree two.

(a) $\rho_{k, l}$ factors through $m$ if and only if $k+l$ is even.

(b) $\rho_{k, l}$ factors through $c$ if and only if $l$ is even.

3. Irreducible representations of monoids. This section contains our main result: the enumeration of all irreducible representations of any normal, irreducible, algebraic monoid $M$ with unit group $S l_{2}(k) \times k^{*}, G l_{2}\left(k^{*}\right)$ or $P G l_{2}(k) \times k^{*}$. Let us first recall the way in which these monoids are classified.

3.1. Theorem [5]. Let $G$ be one of the above groups and let

$$
\mathscr{E}(G)=\{M \mid G(M) \cong G, M \text { is normal and } 0 \in M\} .
$$

There is a canonical one-to-one correspondence

$$
\mathscr{E}(G) \cong \mathbb{Q}^{+},
$$

where $\mathbb{Q}^{+}$denotes the set of positive rational numbers.

For the purposes of this paper we shall need a recipe for the set of characters of the closure $\bar{T}(r)$ in $M_{r}\left(r \in \mathbb{Q}^{+}\right.$, as in 3.1) of a maximal torus $T$ of $G$. The computation may be found in section 4.5 of [5]. Here, $\langle\ldots\rangle$ denotes "submonoid generated by".

3.2. $S l_{2}(k) \times k^{*}$.

$$
X(\bar{T}(r))=\{\chi \in X(T) \mid k \chi \in\langle m a+n b, m a-n b\rangle \text { some } k>0\} .
$$

where $r=n / m,(n, m)=1$. 
3.3. $G l_{2}(k)$.

$X(\bar{T}(r))=\{\chi \in X(T) \mid k \chi \in\langle(m+n) u+(m-n) v,(m+n) v+(m-n) u\rangle$ some $k>0\}$, where $r=n / m,(m, n)=1$.

3.4. $P G l_{2}(k) \times k^{*}$.

$$
X(\bar{T}(r))=\{\chi \in X(T) \mid k \chi \in\langle m x+n y, m x-n y\rangle \text { some } k>0\},
$$

where $r=n / m,(m, n)=1$.

3.5. Lemma. (a) Let $M_{r} \in \mathscr{E}(G), G=S l_{2}(k) \times k^{*}$. Then

$$
W(k, l) \subseteq X(\bar{T}(r)) \text { iff } l / k \leq r .
$$

(b) Let $M_{r} \in \mathscr{E}(G), G=G l_{2}(k)$. Then $W(k, l) \subseteq X(\bar{T}(r))$ iff $2 \mid k+l$ and $l / k \leq r$.

(c) Let $M_{r} \in \mathscr{E}(C), G=P G l_{2}(k) \times k^{*}$. Then $W(k, l) \subseteq X(\bar{T}(r))$ iff $2 \mid l$ and $l / k \leq 2 r$.

Proof. It follows from 3.2 that

$$
X(\bar{T}(r))=\{(k a, l b) \in X(T) \mid-r \leq l / k \leq r\} \cup\{(0,0)\} .
$$

Thus, the conclusion follows from (1) above. For (b) and (c), apply (a) using 2.1.

3.6. Proposition [5]. Let $M \in \mathscr{E}(G)$ and suppose we have morphisms $\alpha: G \rightarrow M^{\prime}$ and $\beta: \bar{T} \rightarrow M^{\prime}$ such that $\left.\alpha\right|_{T}=\left.\beta\right|_{T}$. Then there exists a unique morphism $\rho: M \rightarrow M^{\prime}$ such that $\left.\rho\right|_{G}=\alpha$ and $\left.\rho\right|_{\bar{T}}=\beta$.

Proof (sketch). Let $e \in \bar{T}$ be a maximal idempotent, and let $T(e) \subseteq \bar{T}$ be the unique open submonoid of $\bar{T}$ such that $E(T(e))=\{1, e\}$. Let $B$ and $B^{-}$be the Borel subgroups of $G$ that contain $T$. Then $m: B_{u} \times T(e) \times B_{u}^{-} \rightarrow M, m(x, y, z)=x y z$, is an open embedding with image, say $U$ (the "big cell"). Notice that $U \cap(M \backslash G) \neq \varnothing$. Thus, $\operatorname{codim}(M \backslash(G \cup U), M) \geq 2$ since $M \backslash G$ is irreducible. Define $\rho^{\prime}: U \rightarrow M^{\prime}$ by

$$
\rho^{\prime}(x, y, z)=\alpha(x) \beta(y) \alpha(z) .
$$

Then $\left.\rho^{\prime}\right|_{G \cap U}=\left.\alpha\right|_{G \cap U}$. Thus, $\rho^{\prime}$ can be extended to $G \cup U$. Hence, by Lemma 5.1 of [2], $\rho^{\prime}$ can be extended to $\rho: M \rightarrow M^{\prime}$.

Let $G$ be as above and let $M \in \mathscr{C}(M)$. Then we let

$$
\operatorname{Rep}(M)=\left\{\rho_{k, l} \mid \rho_{k, l} \text { extends to } M\right\},
$$

since any such extension is unique. Recall that these representations are not in general irreducible.

3.7. THEOREM. (a) Let $G=S l_{2}(k) \times k^{*}, M=M_{r}$.

$$
\operatorname{Rep}(M)=\left\{\rho_{k, l} \mid l / k \leq r\right\} .
$$

(b) Let $G=G l_{2}(k), M=M_{r}$.

$$
\operatorname{Rep}(M)=\left\{\rho_{k, l}|l / k \leq r, 2| k+l\right\}
$$


(c) Let $G=P G l_{2}(k) \times k^{*}, M=M_{r}$.

$$
\operatorname{Rep}(M)=\left\{\rho_{k, l}|l / k \leq 2 r, 2| l\right\}
$$

Proof. It will suffice to prove (a). (b) and (c) are similar. If $\rho_{k, l}: G \rightarrow G l_{l+1}(k)$ extends to $M_{r}$ then $W(k, l) \subseteq X(\bar{T}(r))$. So by $3.5, l / k \leq r$.

Conversely, if $l / k \leq r$ then again by $3.5,\left.\rho_{k, l}\right|_{T}$ extends to $\bar{T}(r)$ since $W(k, l) \subseteq$ $X(\bar{T}(r)$ ) (see Lemma 4.1 of [5]). But then 3.6 applies to yield the desired extension $\rho_{k, l}: M \rightarrow \operatorname{End}_{k}\left(k^{l+1}\right)$.

3.8. Scholium. In each case $\operatorname{Rep}\left(M_{r}\right)$ can be identified with a subset of $X(\bar{T}(r))$ :

(a) $\eta: \operatorname{Rep}\left(M_{r}\right) \rightarrow X(\bar{T}(r)), \rho_{k, l} \rightarrow(k, l)$,

(b) $\eta: \operatorname{Rep}\left(M_{r}\right) \rightarrow X(\bar{T}(r)), \rho_{k, l} \rightarrow((k-l) / 2,(k+l) / 2)$,

(c) $\eta: \operatorname{Rep}\left(M_{r}\right) \rightarrow X(\bar{T}(r)), \rho_{k, l} \rightarrow(k, l / 2)$.

3.9. Example. Let $G=G l_{2}(k), M=M(3 / 2) \in \mathscr{E}(G)$.

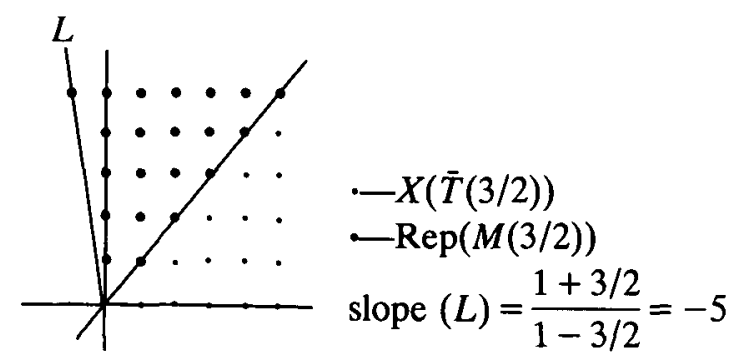

3.10. Remarks. (1) In each case of $3.8, \operatorname{Rep}\left(M_{r}\right)$ contains exactly one element of each $\mathbb{Z}_{2}=N_{G}(T) / T$ orbit of $X(\bar{T}(r))$.

(2) The character $\eta\left(\rho_{k, l}\right)$ of 3.8 is the element of $W(k, l)$ with "highest slope".

3.11. TheOREM. Let $\operatorname{Rep}\left(M_{r}\right)$ be as above. Then there is a canonical one-to-one correspondence

$$
\left\{(\rho, V) \mid \rho \text { is an irreducible representation of } M_{r}\right\} \cong \operatorname{Rep}\left(M_{r}\right) .
$$

In particular, the remarks of 3.10 can be applied to the irreducible representations of $M_{r}$.

Proof. If $\operatorname{char}(k)=0$ then each $\rho_{k, l}$ is irreducible. So assume char $k=p>0$.

Let $S^{l}$ denote the $l$-th symmetric power of $k^{2}$. Given $\rho_{k, l}: M_{r} \rightarrow \operatorname{End}_{k}\left(S^{\prime}\right)$, let $(\rho, V)$ be the irreducible representation of $G$ with highest weight $l$. Assume here, that $G=S l_{2}(k) \times k^{*}$. By $[1, \mathrm{~A} \S 7.5]$

$$
V \cong \pi_{0} \otimes \pi_{1}^{F} \otimes \ldots \otimes \pi_{s}^{F^{s}}=\pi
$$

where each $\pi_{i}$ is infinitesimally irreducible and $\pi_{i}^{F^{i}}$ is obtained from $\pi_{i}$ by composition with the $i$ th power of the Frobenius morphism. Furthermore, $\pi_{i} \cong S^{l_{i}}$ as $S l_{2}(k)$-modules 
for some $0 \leq l_{i}<p$, and the highest weight of $V$ is $l=\sum_{i=0} l_{i} p^{i}$. Hence, we obtain

$$
\pi_{0} \otimes \ldots \otimes \pi_{s}^{F^{s}} \underset{\otimes j_{i}}{\longrightarrow} S^{l_{0}} \otimes \ldots \otimes S^{\mathrm{p}^{l} l_{s}} \underset{\mu}{\longrightarrow} S^{l}
$$

where $j_{i}$ is the inclusion and $\mu$ is the multiplication map, $\mu\left(v_{0} \otimes \ldots \otimes v_{s}\right)=v_{0} \ldots \cdot v_{s}$. Since $\mu \circ\left(\otimes j_{i}\right)$ is nonzero, it is an embedding of $S l_{2}(k)$-modules. Thus, $\pi$ is actually an $M_{r}$-submodule of $S^{l}$ since it is $G\left(M_{r}\right)$-stable and $G\left(M_{r}\right)$ is dense in $M_{r}$.

Conversely, if $\rho: M_{r} \rightarrow \operatorname{End}(V)$ is irreducible, then

(i) $\left.\rho\right|_{k^{*}}=\chi_{k}, \chi_{k}(t)=t^{k}$, some $k$.

(ii) $\left.\rho\right|_{s l_{2}(k)} \cong \pi$ (as representations) for some $\pi$ as above.

But then $\left.\rho\right|_{G}$ is the restriction of $\left.\rho_{k, l}\right|_{G}$ to $\pi \subseteq S^{l}$.

Thus we have

$$
W(k, l) \subseteq X(T)
$$

and

$$
m W(k, l) \subseteq X(\bar{T}), \text { some } m>0,
$$

where $T \subseteq G$ is a maximal torus (i.e. some multiple of each weight of $T$ on $S^{l}$ is contained in the semigroup of weights of $T$ on $\pi$ ). Hence, since $\bar{T}$ is normal [5], $W(k, l) \subseteq X(\bar{T})$. But then 3.5 and 3.7 combine to prove that $\rho_{k, l}$ extends from $G$ to $M_{r}$.

\section{REFERENCES}

1. A. Borel, R. Carter, C. W. Curtis, N. Iwahori, T. A. Springer and R. Steinberg, Seminar on algebraic groups and related finite groups, Lecture Notes in Mathematics 131 (Springer, 1970).

2. F. Grosshans, Observable groups and Hilbert's fourteenth problem. Amer. J. Math. 95 (1973), 229-253.

3. J. Humphreys, Linear algebraic groups, Graduate Texts in Mathematics (Springer, 1981).

4. M. Putcha and L. Renner, The lattice of $J$-classes and the system of idempotents of reductive algebraic monoids, J. Algebra, to appear.

5. L. Renner, Classification of semisimple rank one monoids, Trans. Amer. Math. Soc. 287 (1985), 457-473.

UNIVERSITY OF WESTERN ONTARIO

London, Canada N6A 5B7 